\title{
AISLAMIENTO, CARACTERIZACIÓN Y EVALUACIÓN DE Trichoderma spp. COMO PROMOTOR DE CRECIMIENTO VEGETAL EN PASTURAS DE RAYGRASS (Lolium perenne) Y TRÉBOL BLANCO (Trifolium repens)
}

\author{
ISOLATION, CHARACTERIZATION AND EVALUATION OF Trichoderma spp. AS \\ PROMOTER OF PLANT GROWTH IN PASTURES OF RAYGRASS (Lolium perenne) \\ AND WHITE CLOVER (Trifolium repens)
}

\section{Ramiro Daniel Acurio Vásconez* y Cinthia Karina España Imbaquingo}

\author{
Grupo de Investigación en Biotecnología Aplicada a los Recursos Naturales (BIOARN), Universidad Politécnica Salesiana, Av. \\ Isabela Católica N23-52 y Madrid, Quito, Ecuador. \\ *Autor para correspondencia: racurio@ups.edu.ec
}

Manuscrito recibido el 3 de octubre de 2016. Revisado el 4 de octubre de 2016. Aceptado, el 19 de diciembre de 2016. Publicado el 31 de diciembre de 2016.

\begin{abstract}
Resumen
El género de hongos Trichoderma han sido muy utilizados en agricultura como controladores biológicos gracias a su capacidad para excretar enzimas exógenas que les permiten actuar como biopesticidas. Este hongo tiene la capacidad de crecer en diversos hábitats, y se los encuentra comúnmente en el suelo y en materia en descomposición. Su papel como promotor de crecimiento vegetal se debe a la capacidad de colonizar rápidamente las raíces de la planta protegiéndolas del ataque de fitopatógenos, lo que se traduce en un incremento en el crecimiento y por la exudación de fitohormonas o la solubilización de fosfatos, Fe, Mn, Mg. El objetivo de este trabajo fue aislar cepas nativas de Trichoderma spp. presentes en el suelo de la hacienda "La Alegría" ubicada en el cantón Pedro Moncayo para su posterior evaluación como promotoras de crecimiento vegetal en pasturas de raygrass y trébol blanco. Las cepas aisladas fueron identificadas mediante claves morfológicas como Trichoderma harzianum y Trichoderma viride. El efecto como promotor de crecimiento se evaluó en campo, en potreros previamente establecidos. Se realizaron tres cortes con un periodo de 45 días, después de cada corte se fertilizaron los potreros con soluciones líquidas del hongo a una concentración de $10^{6}$, materia orgánica (Compost $45 \% \mathrm{MO}$ ) y fertilizante químico (Fertiforraje). Los tratamientos que mejores resultados arrojaron fueron T1 con un promedio de materia verde de 12,72 TM/ha/corte y T6 con un promedio de 11,55 $\mathrm{TM} / \mathrm{ha} /$ corte, en comparación con el testigo y el tratamiento químico.
\end{abstract}

Palabras claves: Trichoderma harzianum, Trichoderma viride, promotor de crecimiento, raygrass, trébol blanco. 


\begin{abstract}
The genus Trichoderma fungi have been widely used in agricultre as biological control method due to their capacity to excret exogenous enzymes allowing them to act as a biopesticide. This fungi has the ability to grow in diverse habitats and they are often finded in soil and decaying matter. Its role as a plant growth promoter is due to their ability to rapidly colonize the roots of the plant, protecting them from the attact of phytopathogenic fungi. This proces results in increased growth and exudation of phytohormones or solubilizing phosphates as $\mathrm{Fe}, \mathrm{Mn}, \mathrm{Mg}$. The objective of this study was to isolate native strains of Trichoderma spp. present in the soil of the farm "La Alegría" located in the canton Pedro Moncayo, for subsequent evaluation as promoters of growth in raygrass and white clover. The isolated strains were identified as Trichoderma harzianum and Trichoderma viride. The effect as growth promoter was in previously established pasture fields. We made three cuts with a 45 days period, after each cut we fertilized the pastures with liquid solutions of the fungus at a concentration of $10^{6}$, organic matter (compost $45 \% \mathrm{MO}$ ) and chemical fertilizer (Fertiforraje). The treatments that work the best were the T1 with green matter of 12,72 MT/Ha/cut and T6 with an average of 11,55 MT/ha/cut, compared with the controller and the chemical fertilizer.
\end{abstract}

Keywords: Trichoderma harzianum, Trichoderma viride, growth promoter, raygrass, white clover.

Forma sugerida de citar: Acurio, R. y C. España. 2017. Aislamiento, caracterización y evaluación de Trichoderma spp. como promotor de crecimiento vegetal en pasturas de raygrass (Lolium perenne) y trébol blanco (Trifolium repens). La Granja: Revista de Ciencias de la Vida. Vol. 25(1):53-61. pISSN:1390-3799; eISSN:1390-8596 


\section{Introducción}

En la zona norte de los cantones Cayambe y Pedro Moncayo, en los últimos años la actividad ganadera ha ido ganando mucho espacio, por lo tanto se ha aumentado el uso de suelo para la producción de forraje. Sin embargo, "la producción de pastos y forrajes se enfrenta a diversos problemas como son la baja estabilidad y persistencia, mismos que se encuentran directamente relacionados con factores químicos, físicos y medio ambientales", página 14 de (Méndez, 2010).

La autora (Torres, 2009), señala que las especies forrajeras son las más utilizadas en la alimentación del ganado, esto debido a que su producción es más económica, razón por la cual el forraje se ha convertido en la base de la actividad, por lo que es necesario prestar especial atención en su producción, la cual por ahora es totalmente convencional, especialmente lo relacionado con el uso de fertilizantes.

La mayoría de productores con la finalidad de mejorar la fertilidad del suelo y de esta forma acelerar la producción, así como también para obtener una buena calidad en sus pastos, se han inclinado por el uso de fertilizantes químicos, que si bien es cierto presentan y proporcionan los efectos deseados, a largo plazo ocasionan serios inconvenientes para el ambiente, "causando problemas de salinización en el suelo, factor que constituye un limitante para el rendimiento y calidad de lo que ahí se cultiva, además de los efectos nocivos sobre la salud de las personas", página 15 de (Méndez, 2010).

Una alternativa para mejorar la fertilidad de los suelos dedicados a la producción de forrajes es el uso de hongos del género Trichoderma spp., "las especies de este género son hongos de vida libre, altamente interactivos en las raíces del suelo y ambiente foliar, con una gran capacidad de inactivar exudados originados en las semillas en germinación". Actualmente, está siendo muy utilizado en diversos cultivos debido al excelente trabajo que realiza como controlador biológico, también parece ser que actúa como promotor de crecimiento (Howell, 2002), página 5.

En varios estudios similares de control de patógenos se ha observado que T. harzianum no sólo redujo la severidad de las enfermedades, sino que también indujo la estimulación del crecimiento de las plantas, existiendo reportes en especies herbáceas como lechuga, maíz, tabaco, zapallo, petunia y tomate (Donoso y Lobos, 2008).
Todos los mecanismos de acción de T. harzianum se basan en el principal papel como promotor de crecimiento vegetal que tiene, el cual se manifiesta desde las primeras fases de la plántula, y que le confiere mayores ventajas a la hora del trasplante. $T$. harzianum se asocia a las raíces de la planta proporcionándole un mayor vigor y crecimiento (Galeano, 2008). La presente investigación busco aislar Trichoderma spp., hongo nativo de la zona de estudio, para su posterior caracterización y propagación en el laboratorio. Asimismo, se evaluó en campo el efecto que éste posee sobre el crecimiento del forraje mediante su inoculación en los potreros existentes en el campo.

\section{Materiales y métodos}

\subsection{Ubicación fase de laboratorio}

La fase experimental se llevó a cabo en el laboratorio de microbiología de la Universidad Politécnica Salesiana del CIVABI que se encuentra ubicado en el Distrito Metropolitano de Quito de la provincia de Pichincha, Ecuador.

\subsection{Muestreo del suelo}

Con el objetivo de aislar cepas nativas de Trichoderma spp. se extrajeron muestras de suelo de la hacienda "La Alegría" ubicada en el cantón Pedro Moncayo. Con la ayuda de una barra y una pala limpias, a una profundidad de $10-20 \mathrm{~cm}$, se procedió a tomar submuestras de suelo, las que fueron depositadas en un recipiente plástico limpio para formar así una muestra completa. A continuación se tomó $500 \mathrm{~g}$ de suelo, que fueron colocados en fundas plásticas y etiquetados debidamente para su respectivo procesamiento.

\subsection{Aislamiento y purificación de cepas de Trichoderma}

De cada muestra se tomó $5 \mathrm{~g}$ de suelo que fueron colocados en fundas estériles con $50 \mathrm{ml}$ de solución de cloruro de sodio al $1 \%$. Se agitaron las muestras manualmente por 15 minutos. De cada muestra se procedió a tomar $100 \mu \mathrm{l}$ y se inoculó en cajas Petri con medio Agar Sabouraud Dextrosa. Las cajas fueron selladas con parafilm e incubadas a $26^{\circ} \mathrm{C}$ durante 7 días o hasta el aparecimiento de las colonias de 
Trichoderma. Para la purificación las colonias identificadas como Trichoderma se realizó transferencia directa cajas Petri con medio PDA (Girard, 1964).

\subsection{Mantenimiento de cepas de Trichoder- $m a$}

Para el mantenimiento de las cepas éstas fueron transferidas a tubos con medio PDA. Los tubos fueron sellados con parafilm e incubados a $26^{\circ} \mathrm{C} \mathrm{du-}$ rante 2 a 3 días o hasta observar el aparecimiento de micelio y esporas. Se añadió a los tubos aceite de vaselina, los mismos que fueron sellados con parafilm y colocados en refrigeración (López, 2007).

\subsection{Identificación de microorganismos ais- lados}

La identificación de aislados se realizó mediante reconocimiento de crecimiento de estructuras microscópicas (hifas, esporas y clamidosporas) y macroscópicas (color de micelio, forma de micelio y crecimiento). Las características macro y microscópicas fueron cotejadas con las claves de identificación de géneros de Deuteromycetes (Gams, 1998), para especies de Trichoderma (Girard, 1964) y la clave interactiva de la APS (Samuels, 2006).

\subsection{Multiplicación masiva de Trichoderma}

Se colocaron $50 \mathrm{~g}$ de sustrato (arroz) cocido en recipientes de vidrio, se sellaron y se colocaron en la autoclave durante 10 minutos a $120^{\circ} \mathrm{C}$. Para la inoculación de T. harzianum y T. viride en los sustratos, se requirió de cultivos puros de los hongos, cada recipiente con el sustrato estéril, fue inoculado con 5 $\mathrm{ml}$ de una suspensión de conidios, previamente preparada a una concentración de $10^{8}$ conidios $/ \mathrm{ml}$, finalmente se incubó a $12 \mathrm{~h} \mathrm{luz/oscuridad} \mathrm{a} 26^{\circ} \mathrm{C}$ por 10 días (Sivila, 2013).

\subsection{Fase de campo}

\subsubsection{Instalación del ensayo}

Cada unidad experimental se conformó por una mezcla de Ray Grass Perenne y Trébol Blanco dispuesta en una parcela de 2,5 metros de largo por 1,5 de ancho, $\left(3,75 \mathrm{~m}^{2}\right)$, para el efecto se utilizó flexómetro, piola y estacas. Adicionalmente, para un mejor manejo de la investigación se identificaron en todas las unidades experimentales las características de cada tratamiento.

\subsubsection{Fertilización}

La fertilización se la realizó cinco días después del corte de la pastura y en función de los tratamientos, los periodos de cortes fueron de 35 días y se realizó un total de tres aplicaciones. El sustrato sólido con Trichoderma se diluyó en agua hasta una concentración de $10^{6}$, se aplicó en los potreros mediante una bomba de mochila en forma de drench. Adicionalmente se aplicó materia orgánica (compost con $45 \% \mathrm{MO}$ ) y fertilizante químico sintético (Fertiforraje) para realizar las respectivas comparaciones.

El diseño experimental utilizado fue un DBCA con arreglo factorial $2^{3}+2$ y con tres repeticiones para los factores especie de Trichoderma (viride, harzianum), materia orgánica (aplicación y no aplicación) y fertiforraje (dosis alta de $300 \mathrm{Kg} / \mathrm{Ha}$ y dosis baja de $150 \mathrm{Kg} / \mathrm{ha}$ ).

\subsection{Determinación del rendimiento de ma- teria verde}

Para determinar la producción de materia verde de la mezcla de pastos, de cada una de las unidades experimentales se procedió al corte de $1 \mathrm{~m}^{2}$ con la ayuda de una hoz y de un cuadrante de varilla metálico, posteriormente con una motoguadaña se realizó el corte de igualación del resto de la unidad experimental. La biomasa fue colocada en fundas y pesada para determinar el rendimiento de materia verde.

\subsection{Determinación de materia seca}

De las muestras de pastos se tomaron 2 submuestras de $100 \mathrm{~g}$ cada una y sometidas a una temperatura de $105^{\circ} \mathrm{C}$ por 24 horas para así determinar el rendimiento en materia seca por diferencia de peso.

\section{Resultados y discusión}

\subsection{Identificación macroscópica de $T$. viride}

Las colonias presentaron un crecimiento rápido (5$8 \mathrm{~cm}$ ), el micelio logró cubrir la superficie de los medios de cultivo en 5 días a $26^{\circ} \mathrm{C}$ en Agar papadextrosa. Las colonias al inicio formaron pústulas algodonosas de un color blanco, posteriormente se 
compactaron y esporularon tomando un color verde de textura granular, con conidióforos ramificados visibles alrededor de la pústula. Los aislados no presentaron el olor característico a coco; sin embargo al inverso de las colonias se puede observar visiblemente una coloración marrón- amarillenta, esto debido a la producción de cristales de color amarillo como se muestra en la Figura $1(\mathrm{c}, \mathrm{d})$. Resultados que concuerdan con las descripciones realizadas por (Gams y Bissett, 1998).

\subsection{Identificación microscópica de $T$. viride}

Tal como se muestra en la Figura 1(a,b), los conidióforos mostraron ramas laterales pareadas las mis- mas que forman un ángulo de $90^{\circ}$ con respecto al eje de donde surgen. Las fiálides presentaron una forma alargada, algo anchas en el centro y con un cuello alargado de color hialino. En ocasiones se observó fiálides individuales mientras que en otros casos se las encontró en grupos de 2 a 3 . Las conidias observadas tenían forma ovoidal y a veces globosa, además tenían un color verde claro y los bordes lisos. Las conidias se presentaron incrustadas en las fiálides a manera de un racimo globoso de umbelas bien definidas. Todo lo anteriormente descrito fue comparado con las claves de identificación taxonómicas tanto de (Samuels et al., 1996) como de (Gams y Bissett, 1998) y concuerdan con la especie Trichoderma viride.
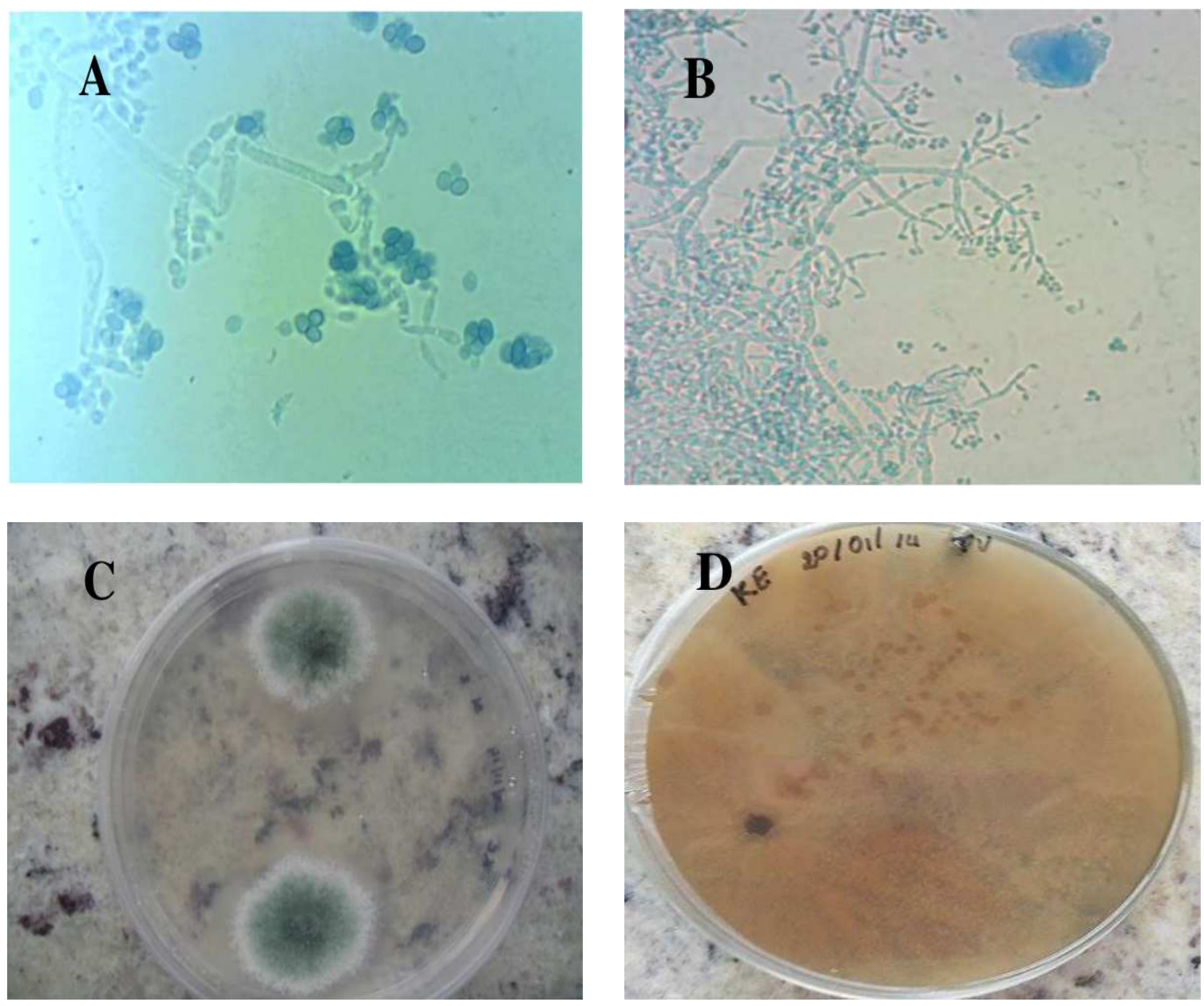

Figura 1. Estructuras microscópicas y macroscópicas de $T$ viride. A) Conidios a los 5 días de incubación. B) Forma de los conidióforos.) C) Formación de anillos concéntricos. D) Pigmentación del medio. 
3.3 Identificación macroscópica de $\boldsymbol{T}$. harzianum

Las colonias tuvieron un radio de 5 a $6 \mathrm{~cm}$, con apariencia aterciopelada en sus etapas tempranas, el micelio presentó un color blanco sin embargo eventualmente fue desarrollando un color blancoverdoso hasta dar una tonalidad verde oliva, además se produjo la formación de anillos concéntricos los mismos que son característicos del género Trichoderma. Después de 7 días de incubación a $25^{\circ} \mathrm{C}$


las colonias presentaron un radio de $9 \mathrm{~cm}$ y cubriendo toda la superficie de la caja Petri y presentaron un color verde obscuro, fueron densas en el centro y se tornaron ondulatorias en los anillos concéntricos hasta llegar a los bordes. Todas las características mencionadas anteriormente fueron comparadas con las descripciones realizadas por Gams y Bissett, (1998), así como también por las descritas por (Samuels et al., 1996), mismas que concuerdan y corresponden a la especie de Trichoderma harzianum y se observan en la Figura 2(c,d).
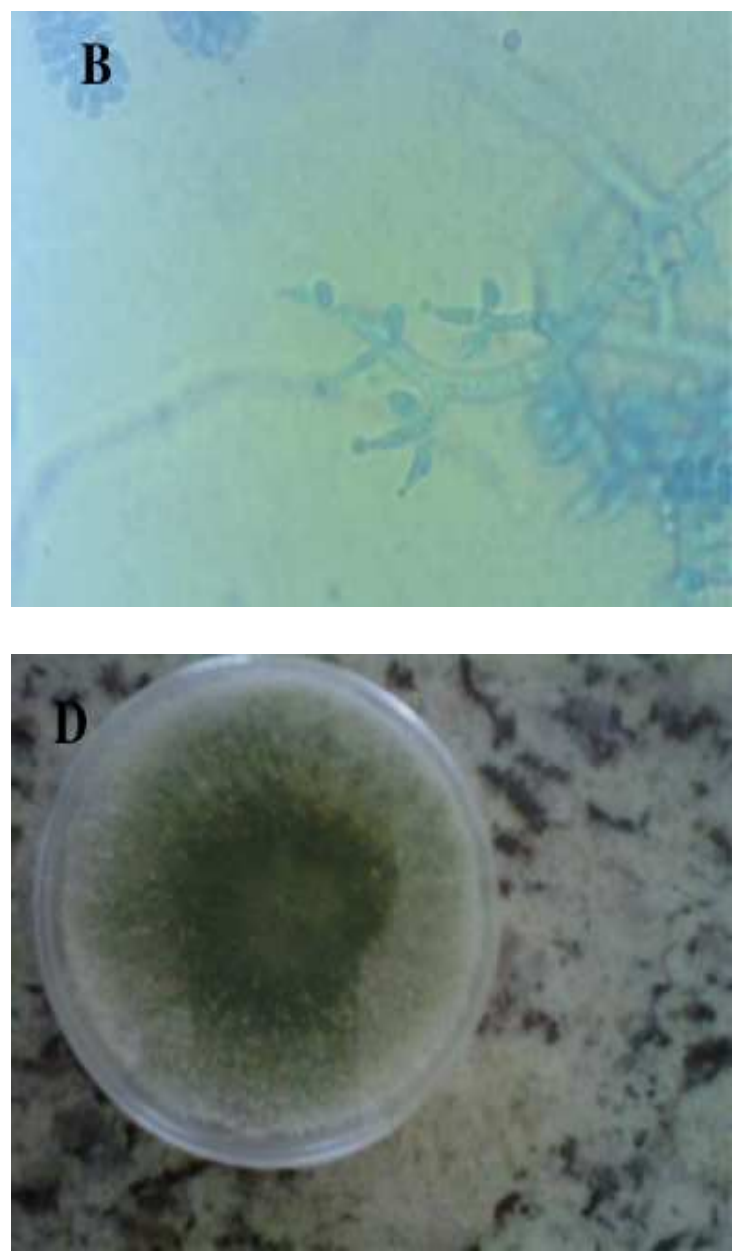

Figura 2. Estructuras microscópicas y macroscópicas de T. harzianum. A y B) Conidióforos a los 5 días de incubación. C) Formación de anillos concéntricos. D) Crecimiento en el medio. 


\subsection{Identificación microscópica de $T$. har- zianum}

Los conidióforos observados a los 5 días de incubación presentaron una ramificación piramidal, "dentro de este sistema de ramificación se puede observar que las ramas o brazos que se encuentran más cercanos al eje principal son más largas"; esta descripción coincide con la hecha por (Samuels et al., 1996), párrafo 1, este mismo autor señala que las ramas que se encuentran más cercanas a la punta forman un ángulo de $90^{\circ}$ con respecto al eje de donde nacen mientras que las ramas que se encuentran más lejanas a la punta forman un ángulo menor a $90^{\circ}$ con respecto al eje de donde surgen. "Las fiálides presentan forma de botella esto se debe a que son más anchas en el centro y se van comprimiendo fuertemente conforme se acercan a la punta hasta formar una especie de cuello".

\subsection{Rendimientos de materia verde y mate- ria seca}

El tratamiento que reportó un mejor desarrollo con un $(\mathrm{p}<0,001)$ durante los tres cortes realizados en la variable producción de materia verde, se presenta en la Figura 3, y fue T1 (Trichoderma harzianum + Materia orgánica + Fertilización alta) con una producción promedio de 12,72 TM/Ha/corte y T6 (Trichoderma viride + Materia orgánica + Fertilización baja). De igual forma T1 mostró el mejor comportamiento para la variable producción de materia seca con un promedio de 1,97 TM/Ha/corte. Estos resultados concuerdan con los obtenidos en diferentes estudios donde se muestran que las cepas de Trichoderma promueven el crecimiento radicular en plantas de maíz y de algunos pastos, además mencionan que "al existir un incremento en la longitud de las raíces colonizadas por Trichoderma, las plantas de maíz y algunos pastos colonizadas por este hongo requirieron un $40 \%$ menos de fertilizantes nitrogenados" (Harman et al., 2004), página 152. Por otro lado existen diversos trabajos donde se asegura que las especies de Trichoderma para poder colonizar las raíces de la planta son capaces sintetizar un sin número de sustancias como péptidos que actúan como promotores de crecimiento, esto es demostrado por Wei Lin y Zhang, (2006), que mediante la aplicación de un proceso de fermentación líquida de Trichoderma harzianum lograron obtener sustancias promoto- ras de crecimiento como ácido indolacético y ácido giberélico, las cuales son una clase de péptidos y son reconocidas como fitohormonas (Wei Lin y Zhang, 2006).

Cabe señalar que el aporte de materia orgánica jugó un papel importante en la producción tanto de materia verde como de materia seca, ya que en los tratamientos donde se agregó materia orgánica se registró un mejor comportamiento en comparación con el testigo y el tratamiento donde solo se utilizó fertilizante químico. La interacción (TH:1:A) presentó la mayor proporción de materia seca en gramos pero no tiene diferencia significativa con (TV:1:B), lo cual demuestra que la aplicación del hongo en presencia de materia orgánica puede reducir la cantidad de fertilizante sintético. Trichoderma tiene la capacidad de degradar la materia orgánica y solubilizar fosfatos orgánicos e inorgánicos mediante mecanismos quelantes y reductores (Cubillos et al., 2009).

En el trabajo realizado por Garcés (2011), se menciona que tanto "la altura como la producción de materia verde en alfalfa, se vieron incrementadas al añadir abono orgánico sólido enriquecido con Trichoderma, los abonos orgánicos presentan un elevado contenido de aminoácidos libres por lo que actúa como activador del desarrollo vegetal", página 76.

\section{Conclusiones}

Dentro de la zona de estudio se logró aislar y caracterizar dos especies, T. harzianum y T. viride. Este hongo favorece el crecimiento de pasturas asociadas de Raygrass y Trébol Blanco aumentando el rendimiento con respecto a la materia verde y materia seca. La incorporación de materia orgánica permite obtener mejores resultados en la absorción de nutrientes y desarrollo de microorganismos en el suelo, evidenciando que $T$. viride puede reducir el uso de fertilizantes de síntesis química cuando se aplica junto con la materia orgánica.

\section{Agradecimiento}

Agradecemos al Ing. Janss Beltrán y la MSc. Nancy Bonifaz que nos facilitaron el trabajo en la hacienda "La Alegría" para el desarrollo de esta investigación. 


\section{Duncan 5\%}

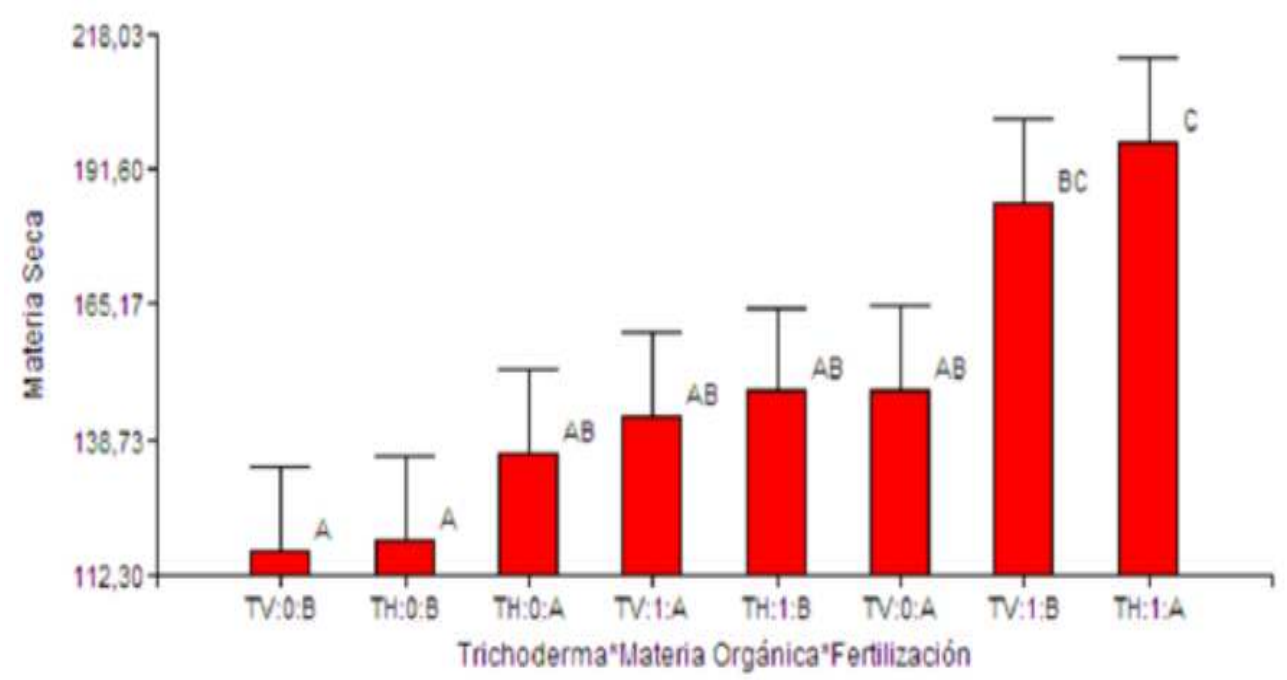

Figura 3. Prueba de Duncan al $5 \%$ para la interacción Trichoderma*Materia orgánica*Fertilización en la variable materia seca (g). TV: T. Viride, TH: T. Harzianum, 0: sin materia orgánica, 1: con materia orgánica, A: Fertilización Alta, B: Fertilización Baja.

\section{Referencias}

Cubillos, J., N. Valero, y L. Mejía. 2009. Trichoderma harzianum como promotor del crecimiento vegetal del maracuyá (Passiflora edulis var. flavicarpa Degener). Agronomia Colombiana. 27(1):81-86. http:/ / www.redalyc.org/articulo.oa?id=1803 14730011

Donoso, E. y G. R. Lobos. 2008. Efecto de Trichoderma harzianum y compost sobre el crecimiento de plántulas de Pinus radiata en vivero. BOSQUE. 29(1):52-57.

Galeano, M. F. 2008. Efecto de Trichoderma harzianum rifai (cepa t-22) sobre cultivos hortícolas. Koppert Biological Systems. Finca Labradorcico del Medio.

Gams, W. y J. Bissett. 1998. Morphology and identification of Trichoderma. Harman GE, Kubicek CP (eds) Trichoderma and Gliocladium. London: Tayler and Francis.

Garcés, S. R. 2011. Evaluación de diferentes niveles de abono orgánico solido potencializado con Trichoderma en la producción de Medicago sativa (Alfalfa) en la estación experimental Sunshi. Riobamba, Chimborazo, Ecuador.
Girard, B. R. 1964. Tecnicas de Microbiologia Agricola. Acribia. Zaragoza-Espana.

Harman, G., C. Howell, A. Viterbo e I. Chet. 2004. Trichoderma species opportunistic avirulent plant symbiots. Nat Rev Microbiol. 2:4356.

Howell, C. 2002. Mechanisms employed by Trichoderma species in the biological control of plant diseases: The history and evolution of current concept. Plant diseae. páginas 4-10.

López, A. 2007. Pruebas de eficiencia in vitro y bajo invernadero de cepas de Trichoderma spp. para el contro de Phytophtora infestans en el cultivo de papa Solanum tuberosum para establecer un banco de microorganismos. Sangolqui-Ecuador.

Mendez, J. 2010. Asturnatura.com. http:/ / www.a sturnatura.com/Lolium perenne

Samuels, G., P. Chaverri, D. Farr y E. McCray. 1996. Trichoderma Online: Systematic Mycology and Microbiology Laboratory, ARS, USDA. (A. U. Systematic Mycology and Microbiology Laboratory, Productor). Trichoderma Online: Systematic Mycology and Microbiology Laboratory, ARS, USDA. 
http://taxadescriptions/keys/TrichodermaI ndex.cfm

Sivila, N. 2013. Producción artesanal de Trichoder$m a$. Tecnologias agroecologicas para la agricultura familiar. Jujuy-Argentina.

Torres, L. A. 2009. Estudio de prefactibilidad para la implementación de la produccion y comercialización de leche cruda en la finca "La Flo- resta". Estudio de prefactibilidad para la implementación de la producción y comercialización de leche cruda en la finca "La Floresta". Quito.

Wei Lin, L. Z.-H. y Z. G.-R. Zhang. 2006. Effects of Peptide in the Fermentation Liquid of Trichoderma harzianum on Nodule Microstructure and Function of Cowpea. Acta Laser Biology Sinica. 84-89. 